Mathematical Modelling and Analysis

Volume 5, 2000, PAGes 164-174

(C) 2000 Technika

\title{
MULTICOMPONENT ITERATIVE METHOD FOR SOLVING TWO-DIMENSIONAL HEAT TRANSFER EQUATION ON MOVING GRIDS
}

\section{S. SYTOVA}

\author{
Institute for Nuclear Problems, Belarus State University \\ Bobruiskaya 11, Minsk, 220050, Republic of Belarus \\ E-mail: sytova@inp.minsk.by
}

Received September 30, 1999

\begin{abstract}
A multicomponent iterative method of domain decomposition on adaptive grids for solution of two-dimensional heat transfer equation is proposed. The adaptive grid is constructed in curvilinear space where Cartesian grid is non-stationary and depends on the solution behavior. In curvilinear space the initial two-dimensional heat transfer equation is converted to the system of nonlinear parabolic equations with mixed derivatives, a source and convective transfer.
\end{abstract}

\section{INTRODUCTION}

The heat transfer equation is one of the basic models of mathematical physics. It can be considered as a test problem for construction of new difference methods for solution of modern scientific problems. Such problems have many dimensions, complicated geometry, different types of nonlinearities.

Numerical and analytical investigations show that application of adaptive grids can increase appreciably the accuracy of numerical algorithms and reduce the grid dimension without the loss of solution accuracy. Adaptive grids allow us to eliminate oscillations, simulated viscosity. Such grids are very effective for solving multidimensional non-stationary problems with domains of strong variation of the solution. The theory of adaptive grids is developed now very intensively [10]-[15].

It was shown [1]-[3] that the multicomponent approach can be effectively 
used for the construction of numerical algorithms. The idea of combination of multicomponent alternating direction method and domain decomposition technique allow us to create a new class of numerical algorithms for different types of problems of mathematical physics, including elliptic and parabolic problems [5]-[9], [16], [17]. These algorithms are unconditionally stable as implicit schemes and are explicit in realization. They have the rate of convergence and computational costs similar to well-known explicit algorithms.

The main idea of this article is to combine adaptive grid technique with multicomponent domain decomposition method for solving a two-dimensional parabolic equation. The adaptive grid is constructed in curvilinear space where the Cartesian grid is non-stationary and moves to domains of strong variation of the solution. It is clear that the full mathematical model has to be supplemented by differential equations which describe the dynamic of Cartesian grid. So, in curvilinear space the full system to be solved is a system of nonlinear parabolic equations with mixed derivatives, a source and convective transfer. Proposed implicit finite difference schemes are nonlinear too. For their effective solution a domain decomposition method is used in the form suggested in [8]. For difference schemes of this method a multicomponent iterative algorithm of decomposition type is proposed. The domain is divided into minimal four-point sub-domains. Presented algorithms allow to obtain independent solution of initial problem in each of sub-domains. This can be important for working on multiprocessor computers. The number of equations which should be solved in such a sub-domain is equal to the number of pattern points. Detailed numerical studies confirmed efficiency of the proposed numerical methods.

\section{STATEMENT OF THE PROBLEM}

In an arbitrary domain $G$ with a boundary $\Gamma$ we consider a two-dimensional parabolic equation :

$$
\begin{aligned}
& \frac{\partial \mathrm{u}}{\partial t}=\frac{\partial}{\partial \mathrm{x}}\left(k \frac{\partial \mathrm{u}}{\partial \mathrm{x}}\right)+\frac{\partial}{\partial \mathrm{y}}\left(k \frac{\partial \mathrm{u}}{\partial \mathrm{y}}\right)+\mathrm{g}, \quad k=k(\mathrm{x}, \mathrm{y})>0, \\
& \mathrm{u}(\mathrm{x}, \mathrm{y}, 0)=\mathrm{u}_{0}(\mathrm{x}, \mathrm{y}), \quad(\mathrm{x}, \mathrm{y}) \in G, \\
& \mathrm{u}(\mathrm{x}, \mathrm{y}, t)=\mathrm{u}_{1}(\mathrm{x}, \mathrm{y}, t), \quad(\mathrm{x}, \mathrm{y}) \in \Gamma, \quad t>0 .
\end{aligned}
$$

After a non-singular coordinates transformation of the general type:

$$
z_{1}=z_{1}(\mathrm{x}, \mathrm{y}, t), \quad z_{2}=z_{2}(\mathrm{x}, \mathrm{y}, t), \quad \tau=t
$$

the domain $G$ is converted to the rectangular domain $\Omega$ (see Fig.1).

Equation (2.1) takes the form:

$$
\frac{\partial(\Psi \mathrm{u})}{\partial \tau}=L \mathrm{u}+I \mathrm{u}+\Psi \mathrm{g}
$$




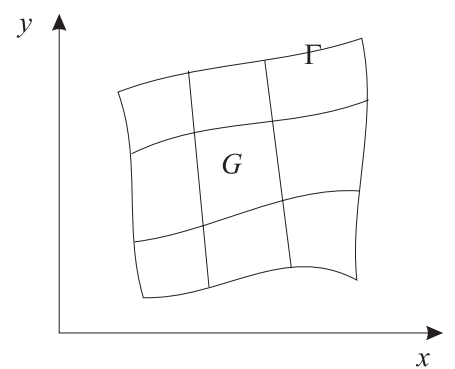

1)

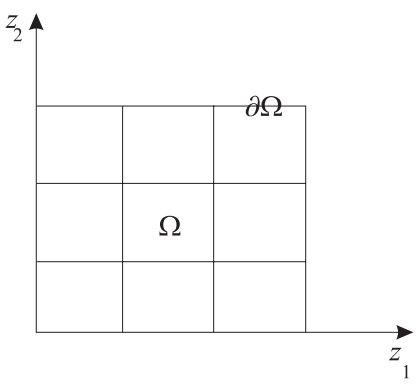

2)

Figure 1. Domain $G$ and domain $\Omega 2$ ) after transformation (2.3).

$$
\begin{gathered}
L \mathrm{u}=\sum_{\alpha, \beta=1}^{2} \frac{\partial}{\partial z_{\alpha}}\left(a_{\alpha \beta} \frac{\partial \mathrm{u}}{\partial z_{\beta}}\right), \quad I \mathrm{u}=\sum_{\alpha=1}^{2} \frac{\partial}{\partial z_{\alpha}}\left(c_{\alpha} \mathrm{u}\right) \\
a_{\alpha \alpha}=\frac{k}{\Psi}\left(\left(\frac{\partial \mathrm{x}}{\partial z_{\alpha}}\right)^{2}+\left(\frac{\partial \mathrm{y}}{\partial z_{\alpha}}\right)^{2}\right), \quad \alpha=1,2 \\
a_{12}=a_{21}=-\frac{k}{\Psi}\left(\frac{\partial \mathrm{x}}{\partial z_{1}} \frac{\partial \mathrm{x}}{\partial z_{2}}+\frac{\partial \mathrm{y}}{\partial z_{1}} \frac{\partial \mathrm{y}}{\partial z_{2}}\right) \\
c_{1}=\frac{\partial \mathrm{x}}{\partial \tau} \frac{\partial \mathrm{y}}{\partial z_{2}}-\frac{\partial \mathrm{y}}{\partial \tau} \frac{\partial \mathrm{x}}{\partial z_{2}}, \quad c_{2}=\frac{\partial \mathrm{y}}{\partial \tau} \frac{\partial \mathrm{x}}{\partial z_{1}}-\frac{\partial \mathrm{x}}{\partial \tau} \frac{\partial \mathrm{y}}{\partial z_{1}} \\
\frac{\partial \mathrm{y}}{\partial \tau}=D_{1} \frac{\partial^{2} \mathrm{y}}{\partial z_{1}^{2}}+D_{2} \frac{\partial^{2} \mathrm{y}}{\partial z_{2}^{2}}+C_{2} \Psi \frac{\partial}{\partial z_{2}}\left(\Psi^{2}\left(\left(\frac{\partial \mathrm{u}}{\partial z_{1}}\right)^{2}+\left(\frac{\partial \mathrm{y}}{\partial z_{2}}\right)^{2}\right)^{1 / 2} \frac{\partial \mathrm{x}}{\partial z_{2}} \frac{\partial \mathrm{y}}{\partial z_{1}},\right. \\
D_{1} \frac{\partial^{2} \mathrm{x}}{\partial z_{1}^{2}}+D_{2} \frac{\partial^{2} \mathrm{x}}{\partial z_{2}^{2}}+C_{1} \Psi \frac{\partial}{\partial z_{1}}\left(\Psi^{2}\left(\left(\frac{\partial \mathrm{u}}{\partial z_{1}}\right)^{2}+\left(\frac{\partial \mathrm{u}}{\partial z_{2}}\right)^{2}\right)^{1 / 2}\right)
\end{gathered}
$$

Initial and boundary conditions (2.2) have the form:

$$
\begin{aligned}
\mathrm{u}\left(z_{1}, z_{2}, 0\right) & =\mathrm{u}_{0}\left(z_{1}, z_{2}\right), \quad\left(z_{1}, z_{2}\right) \in \Omega, \\
\mathrm{u}\left(z_{1}, z_{2}, \tau\right) & =\mathrm{u}_{1}\left(z_{1}, z_{2}, \tau\right), \quad\left(z_{1}, z_{2}\right) \in \partial \Omega, \quad \tau>0 .
\end{aligned}
$$


$D_{1}, D_{2}, C_{1}, C_{2}$ are positive constants of the order of $O(1)$.

Equations (2.6)-(2.7) describe the dynamics of rectangular Cartesian coordinates. They correspond to a grid with nodes that are collected in domain of strong variation of solution. Such form of adaptive grid was suggested in [11]. The type of (2.6) - (2.7) depends on concrete situation. One can take more simple equations that describe quasi-uniform grids [11]:

$$
\frac{\partial \mathrm{x}}{\partial \tau}=D_{1} \frac{\partial^{2} \mathrm{x}}{\partial z_{1}^{2}}+D_{2} \frac{\partial^{2} \mathrm{x}}{\partial z_{2}^{2}}, \quad \frac{\partial \mathrm{y}}{\partial \tau}=D_{1} \frac{\partial^{2} \mathrm{y}}{\partial z_{1}^{2}}+D_{2} \frac{\partial^{2} \mathrm{y}}{\partial z_{2}^{2}} .
$$

Equations (2.6) - (2.7) or (2.9) are equations of the transformation inverse for introduced above (2.3):

$$
\mathrm{x}=\mathrm{x}\left(z_{1}, z_{2}, \tau\right), \quad \mathrm{y}=\mathrm{y}\left(z_{1}, z_{2}, \tau\right), \quad t=\tau .
$$

Since we have supposed that transformation (2.3) is non-singular:

$$
\frac{\partial z_{1}}{\partial \mathrm{x}} \frac{\partial z_{2}}{\partial \mathrm{y}}-\frac{\partial z_{2}}{\partial \mathrm{x}} \frac{\partial z_{1}}{\partial \mathrm{y}}>0
$$

The Jacobian of the inverse transformation $\Psi$ (2.5) will be positive too.

In this case the positive determinacy of the symmetric matrix $A=\left\{a_{\alpha \beta}\right\}$ takes place. And for operator $L$ the strong ellipticity condition takes place:

$$
b_{1} \sum_{\alpha=1}^{2} \xi_{\alpha}^{2} \leq \sum_{\alpha, \beta=1}^{2} a_{\alpha \beta} \xi_{\alpha} \xi_{\beta} \leq b_{2} \sum_{\alpha=1}^{2} \xi_{\alpha}^{2},
$$

where $0<b_{1}<b_{2}$ are bounded constants and $\left(\xi_{1}, \xi_{2}\right)^{T}$ is an arbitrary nonzero real vector.

As a result of transformation (2.3) the initial equation (2.1) is converted to the system of nonlinear parabolic equations with mixed derivatives, a source and convective transfer that is determined by the coefficients $c_{1}, c_{2}$.

Let us transform the equations for adaptive grid (2.6) - (2.7) by differentiating over $z_{1}$ and $z_{2}$ :

$$
\begin{aligned}
& \frac{\partial \mathrm{x}}{\partial \tau}=D_{1} \frac{\partial^{2} \mathrm{x}}{\partial z_{1}^{2}}+D_{2} \frac{\partial^{2} \mathrm{x}}{\partial z_{2}^{2}}+\Phi_{1} \mathrm{u} \\
& \frac{\partial \mathrm{y}}{\partial \tau}=D_{1} \frac{\partial^{2} \mathrm{y}}{\partial z_{1}^{2}}+D_{2} \frac{\partial^{2} \mathrm{y}}{\partial z_{2}^{2}}+\Phi_{2} \mathrm{u}
\end{aligned}
$$

where

$$
\Phi_{1} \mathrm{u}=C_{1} \Psi^{2}\left(2 \varphi_{1}(\mathrm{f}(\mathrm{u}))^{1 / 2}+\Psi(\mathrm{f}(\mathrm{u}))^{-1 / 2}\left(\frac{\partial \mathrm{u}}{\partial z_{1}} \frac{\partial^{2} \mathrm{u}}{\partial z_{1}^{2}}+\frac{\partial \mathrm{u}}{\partial z_{2}} \frac{\partial^{2} \mathrm{u}}{\partial z_{1} \partial z_{2}}\right)\right),
$$




$$
\begin{gathered}
\Phi_{2} \mathrm{u}=C_{2} \Psi^{2}\left(2 \varphi_{2}(\mathrm{f}(\mathrm{u}))^{1 / 2}+\Psi(\mathrm{f}(\mathrm{u}))^{-1 / 2}\left(\frac{\partial \mathrm{u}}{\partial z_{1}} \frac{\partial^{2} \mathrm{u}}{\partial z_{1} \partial z_{2}}+\frac{\partial u}{\partial z_{2}} \frac{\partial^{2} \mathrm{u}}{\partial z_{2}^{2}}\right)\right), \\
\mathrm{f}(\mathrm{u})=\left(\frac{\partial \mathrm{u}}{\partial z_{1}}\right)^{2}+\left(\frac{\partial \mathrm{u}}{\partial z_{2}}\right)^{2}, \\
\varphi_{1}=\frac{\partial \Psi}{\partial z_{1}}=\frac{\partial^{2} \mathrm{x}}{\partial z_{1}^{2}} \frac{\partial \mathrm{y}}{\partial z_{2}}+\frac{\partial x}{\partial z_{1}} \frac{\partial^{2} \mathrm{y}}{\partial z_{1} \partial z_{2}}-\frac{\partial^{2} x}{\partial z_{1} \partial z_{2}} \frac{\partial \mathrm{y}}{\partial z_{1}}-\frac{\partial x}{\partial z_{2}} \frac{\partial^{2} \mathrm{y}}{\partial z_{1}^{2}} \\
\varphi_{2}=\frac{\partial \Psi}{\partial z_{2}}=\frac{\partial^{2} \mathrm{x}}{\partial z_{1} \partial \mathrm{x}_{2}} \frac{\partial \mathrm{y}}{\partial z_{2}}+\frac{\partial \mathrm{x}}{\partial z_{1}} \frac{\partial^{2} \mathrm{y}}{\partial z_{2}^{2}}-\frac{\partial^{2} x}{\partial z_{2}^{2}} \frac{\partial \mathrm{y}}{\partial z_{1}}-\frac{\partial x}{\partial z_{2}} \frac{\partial^{2} \mathrm{y}}{\partial z_{1} \partial z_{2}}
\end{gathered}
$$

\section{FINITE DIFFERENCE SCHEMES}

Let us write down finite difference schemes for nonlinear system $(2.4)-(2.5)$, (2.11) - (2.12). Let us introduce uniform grids in the domain $\Omega$ :

$$
\begin{gathered}
\omega_{h}=\left\{\left(z_{i_{1}}, z_{i_{2}}\right), z_{i_{1}}=i_{1} h_{1}, i_{1}=\overline{0, N_{1}}, N_{1}=\left[L_{1} / h_{1}\right], z_{i_{2}}=i_{2} h_{2},\right. \\
\left.i_{2}=\overline{0, N_{2}}, N_{2}=\left\lfloor L_{2} / h_{2}\right\rfloor\right\}
\end{gathered}
$$

and the time grid: $\omega_{\tau}=\left\{\tau_{j}=j h_{\tau}, j=\overline{0, N_{\tau}}, N_{\tau}=\left\lfloor\mathrm{T} / h_{\tau}\right\rfloor\right\}$.

We use the notation from [14]. The finite difference schemes for adaptive grid equations are given in the form:

$$
\begin{aligned}
& x_{\tau}=D_{1} \widehat{x}_{\bar{z}_{1} z_{1}}+D_{2} \widehat{x}_{\bar{z}_{2} z_{2}}+\Phi_{1} u, \\
& y_{\tau}=D_{1} \widehat{y}_{\bar{z}_{1} z_{1}}+D_{2} \widehat{y}_{\bar{z}_{2} z_{2}}+\Phi_{2} u \\
& \Phi_{1} u=C_{1} \Psi^{2}\left(2 \phi_{1} f^{1 / 2}+\Psi f^{-1 / 2}\left(u_{\check{z}_{1}} u_{\bar{z}_{1} z_{1}}+0.5 u_{\check{z}_{2}}\left(u_{\bar{z}_{1} z_{2}}+u_{z_{1} \bar{z}_{2}}\right)\right)\right), \\
& \Phi_{2} u=C_{2} \Psi^{2}\left(2 \phi_{2} f^{1 / 2}+\Psi f^{-1 / 2}\left(u_{z_{2}} u_{\bar{z}_{2} z_{2}}+0.5 u_{\check{z}_{1}}\left(u_{\bar{z}_{1} z_{2}}+u_{z_{1} \bar{z}_{2}}\right)\right)\right), \\
& \phi_{1}=x_{\bar{z}_{1} z_{1}} y_{\check{z}_{2}}+0.5 x_{\stackrel{\circ}{1}_{1}}\left(y_{\bar{z}_{1} z_{2}}+y_{z_{1} \bar{z}_{2}}\right)-0.5\left(x_{\bar{z}_{1} z_{2}}+x_{z_{1} \bar{z}_{2}}\right) y_{\check{z}_{1}}-x_{\stackrel{\circ}{2}_{2}} y_{\bar{z}_{1} z_{1}}, \\
& \phi_{2}=0.5\left(x_{\bar{z}_{1} z_{2}}+x_{z_{1} \bar{z}_{2}}\right) y_{\mathrm{o}_{2}}+x_{\mathrm{o}_{1}} y_{z_{2} z_{2}}-x_{\bar{z}_{2} z_{2}} y_{\mathrm{z}_{1}}-0.5 x_{\mathrm{z}_{2}}\left(y_{z_{1} z_{2}}+y_{z_{1} \bar{z}_{2}}\right), \\
& f=\left(u_{\check{z}_{1}}\right)^{2}+\left(u_{\mathrm{z}_{2}}\right)^{2} .
\end{aligned}
$$

The following system of difference equations can be written for $(2.4)-(2.5)$ :

$$
(\Psi u)_{\tau}=A \widehat{u}+\widehat{\Psi} \widehat{g}
$$


Multicomponent iterative method for solving 2D heat transfer equation 169

$$
\begin{aligned}
& A u=\frac{1}{2} \sum_{\alpha, \beta=1}^{2}\left(\left(a_{\alpha \beta} u_{\bar{z}_{\beta}}\right)_{z_{\alpha}}+\left(a_{\alpha \beta} u_{z_{\beta}}\right)_{\bar{z}_{\alpha}}\right)+\sum_{\alpha=1}^{2}\left(c_{\alpha} u\right)_{\check{z}_{\alpha}}, \\
& a_{\alpha \alpha}=\frac{k}{\Psi}\left(\left(x_{z_{\beta}}\right)^{2}+\left(y_{z_{\beta}}\right)^{2}\right), \quad \alpha, \beta=1,2, \quad \beta \neq \alpha, \\
& a_{12}=a_{21}=-\frac{k}{\Psi}\left(x_{\check{z}_{1}} x_{\check{z}_{2}}+y_{\check{z}_{1}} y_{\check{z}_{2}}\right),
\end{aligned}
$$

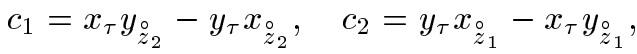

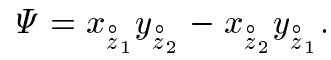

Schemes (3.1) - (3.5) approximate the initial problem (2.4) - (2.5), (2.11) - (2.12) with the order $O\left(h_{\tau}+h_{1}^{2}+h_{2}^{2}\right)$. Positivity of the operators in equations (2.4)-(2.5), (2.11)-(2.12) guarantees the stability and the convergence of difference schemes (3.1)-(3.5). This can be proved by the energy inequalities method [14].

\section{DOMAIN DECOMPOSITION METHOD}

In order to solve nonlinear system (3.1)-(3.5) we use a domain decomposition method in the form [8].

Let us break up the domain $\Omega$ into minimal sub-domains $\omega_{i_{1} i_{2}}=\left\{\left(z_{i_{1}}, z_{i_{2}}\right)\right.$, $\left.\left(z_{i_{1}}, z_{i_{2}+1}\right),\left(z_{i_{1}+1}, z_{i_{2}+1}\right),\left(z_{i_{1}+1}, z_{i_{2}}\right)\right\}$ (see Fig. 2). In each of four points of a sub-domain we define four values of grid functions:

$$
\begin{aligned}
& x_{i_{1} i_{2}}=\left(x_{i_{1} i_{2}}^{(0)}, \ldots, x_{i_{1} i_{2}}^{(3)}\right) \quad y_{i_{1} i_{2}}=\left(y_{i_{1} i_{2}}^{(0)}, \ldots, y_{i_{1} i_{2}}^{(3)}\right), u_{i_{1} i_{2}}=\left(u_{i_{1} i_{2}}^{(0)}, \ldots, u_{i_{1} i_{2}}^{(3)}\right) \text {. } \\
& \begin{array}{lll|lll|lll}
1 & & 2 & 1 & & 2 & 1 & & 2 \\
& \omega_{i_{1}-1, i_{2}+1} & & & \omega_{i_{1}, i_{2}+1} & & & \omega_{i_{1}+1, i_{2}+1} & \\
0 & & 3 & 0 & & 3 & 0 & & 3 \\
\hline 1 & & 2 & 1 & & 2 & 1 & & 2 \\
& \omega_{i_{1}-1, i_{2}} & & \omega_{i_{1}, i_{2}} & & & \omega_{i_{1}+1, i_{2}} & \\
0 & & 3 & 0 & & 3 & 0 & & 3 \\
\hline 1 & & 2 & 1 & & 2 & 1 & & 2 \\
& \omega_{i_{1}-1, i_{2}-1} & & \omega_{i_{1}, i_{2}-1} & & & \omega_{i_{1}+1, i_{2}-1} & \\
0 & & 3 & & & 3 & 0 & & 3
\end{array}
\end{aligned}
$$

Figure 2. Fragment of the grid $\omega_{h}$.

Let us consider the equation for the space coordinate $x(3.1)$ :

$$
x_{\tau}=A_{x} \widehat{x}+\Phi_{1} u,
$$


where operator $A_{x}$ has the form:

$$
A_{x} x=D_{1} \widehat{x}_{\bar{z}_{1} z_{1}}+D_{2} \widehat{x}_{\bar{z}_{2} z_{2}} .
$$

In each grid point $\left(z_{i_{1}}, z_{i_{2}}\right)$ it is represented by the sum of four operators:

$$
A_{x} x=\sum_{\alpha=0}^{3} A_{x}^{(\alpha)} x .
$$

Operators $A_{x}^{(\alpha)}, \alpha=\overline{0,3}$, have the form:

$$
\begin{gathered}
A_{x}^{(0)} x_{i_{1}, i_{2}}=0.5 \sum_{\alpha=1}^{2} \Upsilon_{\alpha}^{+} x_{i_{1}, i_{2}}^{(0)}, \quad A_{x}^{(1)} x_{i_{1}, i_{2}}=0.5\left(\Upsilon_{1}^{+} x_{i_{1}, i_{2}}^{(1)}+\Upsilon_{2}^{-} x_{i_{1}, i_{2}}^{(1)}\right) \\
A_{x}^{(2)} x_{i_{1}, i_{2}}=0.5 \sum_{\alpha=1}^{2} \Upsilon_{\alpha}^{-} x_{i_{1}, i_{2}}^{(2)}, \quad A_{x}^{(3)} x_{i_{1}, i_{2}}=0.5\left(\Upsilon_{1}^{-} x_{i_{1}, i_{2}}^{(3)}+\Upsilon_{2}^{+} x_{i_{1}, i_{2}}^{(3)}\right) \\
\Upsilon_{1}^{+} x=h_{1}^{-1} D_{1} x_{z_{1}}, \quad \Upsilon_{2}^{+} x=h_{2}^{-1} D_{2} x_{z_{2}}, \\
\Upsilon_{1}^{-} x=-h_{1}^{-1} D_{1} x_{\bar{z}_{1}}, \quad \Upsilon_{2}^{-} x=-h_{2}^{-1} D_{2} x_{\bar{z}_{2}} .
\end{gathered}
$$

We repeat the same operations for the equation (3.2):

$$
y_{\tau}=A_{y} \widehat{y}+\Phi_{2} u \text {. }
$$

Since the operator $A_{y}=A_{x}$, so it has the form (4.2)-(4.3).

Let us introduce the following designations:

$$
\begin{gathered}
\tilde{v}_{i_{1}, i_{2}}=\left(\tilde{v}_{i_{1}, i_{2}}^{(0)}, \ldots, \tilde{v}_{i_{1}, i_{2}}^{(3)}\right), \\
\tilde{v}_{i_{1}, i_{2}}^{(0)}=0.25\left(v_{i_{1}, i_{2}}^{(0)}+v_{i_{1}, i_{2}-1}^{(1)}+v_{i_{1}-1, i_{2}-1}^{(2)}+v_{i_{1}-1, i_{2}}^{(3)}\right), \\
\tilde{v}_{i_{1}, i_{2}}^{(1)}=0.25\left(v_{i_{1}, i_{2}}^{(1)}+v_{i_{1}-1, i_{2}}^{(2)}+v_{i_{1}-1, i_{2}+1}^{(3)}+v_{i_{1}, i_{2}+1}^{(0)}\right), \\
\tilde{v}_{i_{1}, i_{2}}^{(2)}=0.25\left(v_{i_{1}, i_{2}}^{(2)}+v_{i_{1}, i_{2}+1}^{(3)}+v_{i_{1}+1, i_{2}+1}^{(0)}+v_{i_{1}+1, i_{2}}^{(1)}\right), \\
\tilde{v}_{i_{1}, i_{2}}^{(3)}=0.25\left(v_{i_{1}, i_{2}}^{(3)}+v_{i_{1}+1, i_{2}}^{(0)}+v_{i_{1}+1, i_{2}-1}^{(1)}+v_{i_{1}, i_{2}-1}^{(2)}\right) .
\end{gathered}
$$

Let us write down the difference scheme of domain decomposition for equation (4.1) in the domain $\omega_{i_{1} i_{2}}$ :

$$
\left(\widehat{x}_{i_{1}, i_{2}}-\widetilde{x}_{i_{1}, i_{2}}\right) / h_{\tau}+A_{x i_{1}, i_{2}}(\widehat{x}-x)+0.25 B_{x_{i_{1}, i_{2}}}(x)=\Phi_{1} \widetilde{u}_{i_{1}, i_{2}},
$$


where

$$
\begin{gathered}
A_{x i_{1}, i_{2}}(x)=\left(-A_{x}^{(0)} x_{i_{1}, i_{2}}, \ldots,-A_{x}^{(3)} x_{i_{1}, i_{2}}\right), \\
B_{x i_{1}, i_{2}}(x)=\left(-B_{i_{1}, i_{2}}^{(0)}(x), \ldots,-B_{i_{1}, i_{2}}^{(3)}(x)\right), \\
B_{i_{1}, i_{2}}^{(0)}(x)=A_{x}^{(0)} x_{i_{1}, i_{2}}^{(0)}+A_{x}^{(1)} x_{i_{1}, i_{2}-1}^{(1)}+A_{x}^{(2)} x_{i_{1}-1, i_{2}-1}^{(2)}+A_{x}^{(3)} x_{i_{1}-1, i_{2}}^{(3)}, \\
B_{i_{1}, i_{2}}^{(1)}(x)=A_{x}^{(1)} x_{i_{1}, i_{2}}^{(1)}+A_{x}^{(2)} x_{i_{1}-1, i_{2}}^{(2)}+A_{x}^{(3)} x_{i_{1}-1, i_{2}+1}^{(3)}+A_{x}^{(0)} x_{i_{1}, i_{2}+1}^{(0)}, \\
B_{i_{1}, i_{2}}^{(2)}(x)=A_{x}^{(2)} x_{i_{1}, i_{2}}^{(2)}+A_{x}^{(3)} x_{i_{1}, i_{2}+1}^{(3)}+A_{x}^{(0)} x_{i_{1}+1, i_{2}+1}^{(0)}+A_{x}^{(1)} x_{i_{1}+1, i_{2}}^{(1)}, \\
B_{i_{1}, i_{2}}^{(3)}(x)=A_{x}^{(3)} x_{i_{1}, i_{2}}^{(3)}+A_{x}^{(0)} x_{i_{1}+1, i_{2}}^{(0)}+A_{x}^{(1)} x_{i_{1}+1, i_{2}-1}^{(1)}+A_{x}^{(2)} x_{i_{1}, i_{2}-1}^{(2)} . \\
\Phi_{1} \tilde{u}_{1, i_{2}}=\left(\Phi_{1} \widetilde{u}_{i_{1}, i_{2}}^{(0)}, \ldots, \Phi_{1} \tilde{u}_{i_{1}, i_{2}}^{(3)}\right)=\left(\Phi_{1}^{(0)}, \ldots, \Phi_{1}^{(3)}\right) .
\end{gathered}
$$

Corresponding iterative algorithm of domain decomposition looks like this:

$$
\left(\stackrel{s+1}{x}_{i_{1}, i_{2}}-\widetilde{x}_{i_{1}, i_{2}}\right) / h_{\tau}+A_{x i_{1}, i_{2}}(\stackrel{s+1}{x}-\stackrel{s}{x})+0.25 B_{x i_{1}, i_{2}}(\stackrel{s}{x})=\Phi_{1} \tilde{u}_{i_{1}, i_{2}}
$$

where ${\stackrel{0}{i_{1}, i_{2}}}_{x_{1_{1}, i_{2}}}$.

Analogous difference scheme of domain decomposition and iterative process can be written for the difference equation (3.2).

Let us consider the equation (3.3). Operator $A$ can be represented in an analogous to (4.2) way. Operators $A^{(\alpha)}, \alpha=\overline{0,3}$ have the following form:

$$
\begin{gathered}
A^{(0)} u_{i_{1}, i_{2}}=M^{(0)} u_{i_{1}, i_{2}}+\Pi^{(0)} u_{i_{1}, i_{2}}, \quad A^{(1)} u_{i_{1}, i_{2}-1}=M^{(1)} u_{i_{1}, i_{2}-1}+\Pi^{(1)} u_{i_{1}, i_{2}-1} \\
A^{(2)} u_{i_{1}-1, i_{2}-1}=M^{(2)} u_{i_{1}-1, i_{2}-1}+\Pi^{(2)} u_{i_{1}-1, i_{2}-1} \\
A^{(3)} u_{i_{1}-1, i_{2}}=M^{(3)} u_{i_{1}-1, i_{2}}+\Pi^{(3)} u_{i_{1}-1, i_{2}} \\
M^{(0)} u=0.5 \sum_{\alpha=1}^{2} \Lambda_{\alpha}^{+} u, \quad M^{(1)} u=0.5\left(\Lambda_{1}^{+} u+\Lambda_{2}^{-} u\right) \\
M^{(2)} u=0.5 \sum_{\alpha=1}^{2} \Lambda_{\alpha}^{-} u, \quad M^{(3)} u=0.5\left(\Lambda_{1}^{-} u+\Lambda_{2}^{+} u\right) \\
\Lambda_{\alpha}^{+} u=h_{\alpha}^{-1} a_{\alpha \alpha} u_{z_{\alpha}}-h_{\alpha}^{-1} c_{\alpha}^{\left.(+1)_{\alpha}\right)} u^{\left(+1_{\alpha}\right)} \\
\Lambda_{\alpha}^{-} u=-h_{\alpha}^{-1} a_{\alpha \alpha}^{\left(-1_{\alpha}\right)} u_{\bar{z}_{\alpha}}+h_{\alpha}^{-1} c_{\alpha}^{\left(-1_{\alpha}\right)} u^{\left(-1_{\alpha}\right)}, \quad \alpha=1,2 \\
\Pi^{(0)} u=0.5\left(h_{1}^{-1} a_{21} u_{z_{2}}+h_{2}^{-1} a_{12} u_{z_{1}}\right)
\end{gathered}
$$




$$
\begin{gathered}
\Pi^{(1)} u=0.5\left(-h_{2}^{-1} a_{21}^{\left(-1_{2}\right)} u_{z_{1}}^{\left(-1_{2}\right)}+h_{1}^{-1} a_{12}^{\left(+1_{1}\right)} u_{\bar{z}_{2}}^{\left(+1_{1}\right)}\right), \\
\Pi^{(2)} u=-0.5\left(h_{2}^{-1} a_{21} u_{\bar{z}_{1}}+h_{1}^{-1} a_{12} u_{\bar{z}_{2}}\right), \\
\Pi^{(3)} u=0.5\left(h_{2}^{-1} a_{12}^{\left(+1_{2}\right)} u_{\bar{z}_{1}}^{\left(+1_{2}\right)}-h_{1}^{-1} a_{21}^{\left(-1_{1}\right)} u_{z_{2}}^{\left(-1_{1}\right)}\right) .
\end{gathered}
$$

The difference scheme of domain decomposition for equation (3.3) in the domain $\omega_{i_{1}, i_{2}}$ can be written as:

$$
\left((\widehat{\Psi} \widehat{u})_{i_{1}, i_{2}}-(\tilde{\Psi} \tilde{u})_{i_{1}, i_{2}}\right) / h_{\tau}+A_{i_{1}, i_{2}}(\widehat{u}-u)+0.25 R_{i_{1}, i_{2}}(u)=\widehat{\Psi} \widehat{g}
$$

where

$$
\begin{gathered}
A_{i_{1}, i_{2}}(u)=\left(-A^{(0)} u_{i_{1}, i_{2}}, \ldots,-A^{(3)} u_{i_{1}, i_{2}}\right), \\
R_{i_{1}, i_{2}}(u)=\left(-R_{i_{1}, i_{2}}^{(0)}(u), \ldots,-R_{i_{1}, i_{2}}^{(3)}(u)\right), \\
R_{i_{1}, i_{2}}^{(0)}(u)=A^{(0)} u_{i_{1}, i_{2}}^{(0)}+A^{(1)} u_{i_{1}, i_{2}-1}^{(1)}+A^{(2)} u_{i_{1}-1, i_{2}-1}^{(2)}+A^{(3)} u_{i_{1}-1, i_{2}}^{(3)}, \\
R_{i_{1}, i_{2}}^{(1)}(u)=A^{(1)} u_{i_{1}, i_{2}}^{(1)}+A^{(2)} u_{i_{1}-1, i_{2}}^{(2)}+A^{(3)} u_{i_{1}-1, i_{2}+1}^{(3)}+A^{(0)} u_{i_{1}, i_{2}+1}^{(0)}, \\
R_{i_{1}, i_{2}}^{(2)}(u)=A^{(2)} u_{i_{1}, i_{2}}^{(2)}+A^{(3)} u_{i_{1}, i_{2}+1}^{(3)}+A^{(0)} u_{i_{1}+1, i_{2}+1}^{(0)}+A^{(1)} u_{i_{1}+1, i_{2}}^{(1)}, \\
R_{i_{1}, i_{2}}^{(3)}(u)=A^{(3)} u_{i_{1}, i_{2}}^{(3)}+A^{(0)} u_{i_{1}+1, i_{2}}^{(0)}+A^{(1)} u_{i_{1}+1, i_{2}-1}^{(1)}+A^{(2)} u_{i_{1}, i_{2}-1}^{(2)} .
\end{gathered}
$$

The iterative algorithm for equation (3.3) can be formulated as:

$$
\left(\left(\widehat{\Psi}^{\stackrel{s+1}{u}}\right)_{i_{1}, i_{2}}-(\tilde{\Psi} \tilde{u})_{i_{1}, i_{2}}\right) / h_{\tau}+A_{i_{1}, i_{2}}(\stackrel{s+1}{u}-\stackrel{s}{u})+0.25 R_{i_{1}, i_{2}}(\stackrel{s}{u})=\widehat{\Psi} \widehat{g}
$$

where ${\stackrel{0}{u_{1}, i_{2}}}=\tilde{u}_{i_{1}, i_{2}}$.

The stability relative to initial data and also the convergence of the difference problem solution (4.4), (4.7) and the iterative algorithms (4.5), (4.8) were investigated in linearized case. We proved that difference schemes (4.4) and (4.7) were unconditionally stable relative to the initial data and converged. Iterative methods (4.5) and (4.8) converge too. It seems to be impossible to examine the full nonlinear system. But since all our operators are positive definite, we can suppose that the stability and the convergence take place. This was confirmed by numerical experiments. We considered a problem of two-dimensional flow of incompressible viscous fluid as a test problem. A next article will be devoted to the discussion of results of numerical experiments carried out.

Author is indebted to V.N. Abrashin for attention to this work and valuable remarks. 
Multicomponent iterative method for solving 2D heat transfer equation 173

\section{REFERENCES}

[1] V.N. Abrashin. On a variant of alternating direction method for solving multidimensional problems of mathematical physics. I. Differential Equations, 26 (7), 1990, 314-323.

[2] V.N. Abrashin. On an economical iterative methods for solving multidimensional problems of mathematical physics. I. Differential Equations, 29 (7), 1993, 1107-1116.

[3] V.N. Abrashin. On a class of iterative methods for solving multidimensional problems of mathematical physics. Differential Equations, 31 (5), 1995, 840-848.

[4] V.N. Abrashin. On a method of domain decomposition when solving problems of mathematical physics. Differential Equations, 32 (5), 1996, 652-660.

[5] V. N. Abrashin. On an iterative method of domain decomposition for solving problems for elliptic equations. I. Differential Equations, 33 (7), 1997, 899-908.

[6] V. N. Abrashin and N.G. Zhadaeva. Multicomponent alternating direction method for solving stationary problems of mathematical physics. I. Differential Equations, 32 (9), 1996, 1212-1221.

[7] V. N. Abrashin and N.G. Zhadaeva. Multicomponent alternating direction method for solving stationary problems of mathematical physics. II. Differential Equations, 33 (9), 1997, 1211-1219.

[8] V. N. Abrashin. On an iterative method of solving difference problems for elliptic equations. Differential Equations, 34 (7), 1998, 911-920.

[9] V.N. Abrashin. On stability of difference schemes of multicomponent alternating direction method for parabolic equations and systems. Differential Equations, 35 (2), 1999, 266-271.

[10] I. Babuska, A. Miller and M. Vogelius. Adaptive method and error estimation for elliptic problems of structural mechanics. Adaptive Comput. Methods for Partial Different. Equations, SIAM, Philadelphia, 1983, 57-79.

[11] N.A. Dari'in and V.I. Mazhukin. Mathematical modeling of nonstationary twodimensional boundary value problem on grids with dynamical adaptation. Mat. Modelirovanie, 1, 1989, 29-43.(In Russian)

[12] W. Hacksburg. From classical numerical mathematics to scientific computing. Documenta Mathematica. Extra Volume ICM, I, 1998, 235-254.

[13] V.D. Liseikin. Survei of methods of structural adaptive grids construction. Zh. Vych. Mat. i Mat. Fiz., 36 (1), 1996, 3-41.

[14] A.A. Samarskii. Theory of difference schemes. Nauka, Moscow, 1989. (In Russian)

[15] J.F. Thompson. Numerical grid generation. North-Hollond, Amstardam, 1992.

[16] N.G. Zhadaeva and E.A. Samarskaya. A method of domain decomposition for solving grid parabolic problems. Differential Equations, 35 (2), 1999, 225-231.

[17] V.M. Volkov and S.N. Lechtikov. Multicomponent iterative methods of decomposition type for two-dimensional stationary problems of dissipative transfer. Differential Equations, 33 (7), 1997, 927-933. 


\title{
DVIMATE்S ŠILUMOS LAIDUMO LYGTIES SPRENDIMAS JUDANC̈IUOSE TINKLUOSE DAUGIAKOMPONENTINIU ITERACINIU METODU
}

\author{
S. SYTOVA
}

Darbe nagrinëjamas daugiakomponentinis iteracinis metodas, kuriuo sprendžiamas dvimatis šilumos laidumo uždavinys. Naudojamas adaptyvus diskretusis tinklas ir skaičiavimo sritis skaidoma $i$ atskiras nepriklausomas dalis. Adaptyvusis tinklas konstruojamas kreivinèse koordinatèse ir jis priklauso nuo uždavinio sprendinio. Po transformacijos gaunama netiesiniu skirtumų lygčiu sistema tenkinanti paraboliškumo sąlygą. Lygtyje atsiranda nariai su mišriomis išvestinèmis, konvekcijos nariai ir šilumos šaltiniai. Gautoji lygčiu sistema sprendžiama panaudojant srities skaidymo ir daugiakomponentinių iteracinių metodų kombinaciją. 